\section{NIKing BAFF's limelight}

[NIK
inhibition] was
superior to
BAFF blockade
in reducing
autoantibody
production

New findings suggest that inhibiting nuclear factor- $\mathrm{kB}$ (NF- $\mathrm{kB}$ ) inducing kinase (NIK) could be as therapeutically efficacious as blocking $B$ cell activating factor (BAFF; also known as TNF ligand superfamily member 13B) in systemic lupus erythematosus (SLE). Researchers at Genentech have developed a potent and selective inhibitor of NIK (NIK SMI1) that ameliorated disease in two mouse models of SLE.

NIK mediates non-canonical NF- $\kappa B$ signalling downstream of

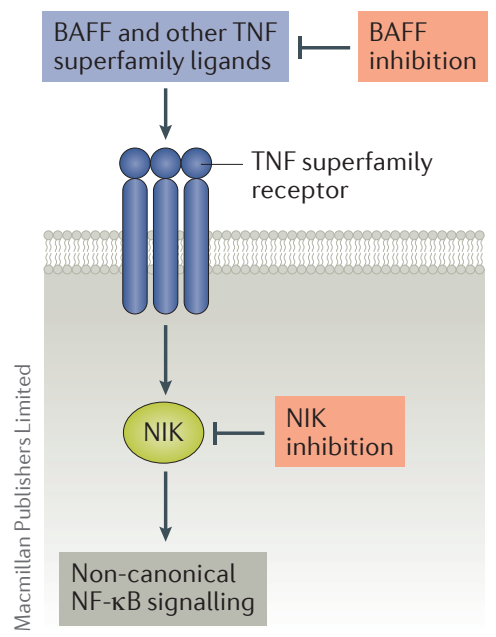

TNF receptor superfamily members. "Given that BAFF primarily signals through the non-canonical NF- $\kappa B$ pathway, a NIK inhibitor should impact the same biology as BAFF blockade does," explains corresponding author Nico Ghilardi. "Many other disease-relevant TNF family members also activate the noncanonical NF- $\kappa$ B pathway. We expected to get added efficacy through partial inhibition of their signalling, and hypothesized that a NIK inhibitor would be a very attractive drug candidate."

In vitro, NIK SMI1 inhibited BAFF-induced and CD40-induced p52 processing in human and mouse $B$ cells, as well as B cell survival. NIK SMI1 treatment also inhibited OX40-induced hyper-proliferation of activated $\mathrm{CD} 4^{+}$memory $\mathrm{T}$ cells, and TNF-related weak inducer of apoptosis (TWEAK)-induced gene expression in human and mouse renal proximal tubulointerstitial epithelial cells, including that of pro-inflammatory genes associated with SLE, indicating that this compound has broad disease-relevant immunosuppressive effects in various cell types.
In vivo, NIK inhibition recapitulated the effects of BAFF blockade in mice, affecting various B cell-dependent end points, such as splenic B cell numbers. Unlike BAFF inhibition, NIK inhibition also affected the $\mathrm{T}$ cell component of the autoantibody response and was superior to BAFF blockade in reducing autoantibody production. In two mouse models of SLE - an IFNa-accelerated model in lupusprone mice and a model in which lupus is induced following repeated exposure to a Toll-like receptor 7 (TLR7) agonist - NIK SMI1 treatment improved survival and reduced renal pathology and proteinuria scores compared with mice treated with vehicle.

"We are currently using NIK SMI1 to interrogate the in vivo biology of NIK in other disease models, so as to further increase the appeal of this potential therapeutic intervention," says Ghilardi.

Jessica McHugh

ORIGINAL ARTICLE Brightbill, H. D. et al. NF-kB inducing kinase is a therapeutic target for systemic lupus erythematosus. Nat. Commun. 9, 179 (2018) 